\title{
The EOD Sound Response in Weakly Electric Fish
}

\author{
Bernd Kramer*, Jürgen Tautz**, and Hubert Markl \\ Universität Konstanz, Fakultät für Biologie, Postfach 5560, D-7750 Konstanz, Federal Republic of Germany
}

Accepted May 8, 1981

Summary. 1. A spontaneous EOD response to sound is described in two gymnotoids of the pulse Electric Organ Discharge (EOD) type, Hypopomus and Gymnotus, and in one mormyrid, Brienomyrus (Figs. 2-4).

2. In all three species, the EOD response to the sound onset was a transient EOD rate increase. In the low EOD rate Hypopomus (3-6 EODs/s at rest) the first, second, or third EOD interval following sound onset was significantly shorter than the average EOD interval before stimulation. The shortest latency found was $100 \mathrm{~ms}$, the longest ca. $1.2 \mathrm{~s}$. Gymnotus (around $50 \mathrm{EODs} / \mathrm{s}$ at rest) responded similarly, but the third interval after sound onset was the first to be affected even at highest intensities (shortest latencies approx. $60 \mathrm{~ms}$; latencies $>0.5 \mathrm{~s}$ at low sound intensities). In Brienomyrus (4-8 EODs/s at rest) the response occurred already at the first EOD interval after sound onset.

3. An EOD sound response was recorded in Hypopomus and in Gymnotus up to $5,000 \mathrm{~Hz}$ sound frequency (in one Gymnotus individual: up to 7,000 Hz). Due to technical limitations the low frequency limit of the response could not be exactly determined: the fishes responded well even below $100 \mathrm{~Hz}$. Hypopomus had its maximum sensitivity around $500 \mathrm{~Hz}$ (Fig. 5), Gymnotus around 1,000 Hz (Fig. 6).

4. In all three species the EOD sound response was graded with sound intensity (Hypopomus: Fig. 7).

5. No EOD response to sound was found in two gymnotoids of the wave type, Eigenmannia and Apteronotus, and in the gymnotoid pulse fish Rhamphichthys. A criterion is proposed by which it should be possible to predict whether or not a weakly electric fish species will show the EOD sound response.

\footnotetext{
* New address: Fakultät für Biologie und Vorklinische Medizin, Zoologisches Institut der Universität, D-8400 Regensburg, Federal Republic of Germany

** Present address: Department of Psychology, Stanford University, Stanford, California 94305, USA
}

6. It is concluded that the EOD response to sound is similar to EOD responses to other kinds of stimulation (light, touch, vibration, food, and even electrical). The possible biological function is discussed.

\section{Introduction}

Conditioning techniques have been widely applied to study the hearing capacities of fish. Spontaneous responses to sound have apparently not been used in modern investigations of fish hearing as these are often unreliable, or difficult to interpret (reviews in Tavolga 1971, 1976; Popper and Fay 1973; Hawkins 1973; Schwartzkopff 1976).

Mormyrid and some gymnotoid weakly electric fish have long been known to display spontaneous Electric Organ Discharge (EOD) rate changes in response to mechanical stimulation (such as tapping the wall of their aquarium: Lissmann 1958, 1961). Monnier's question (in Lissmann 1961) whether "any correlation in electric fish between their electrical activity and the sensitivity to sound" had been found remained apparently unanswered up to now.

This is surprising since the hearing capacities of two mormyrids as well as of one gymnotoid have already been investigated by von Frisch and his coworkers (von Frisch 1936, 1938; Diesselhorst 1938; Stipetic 1939; von Boutteville 1935) by conditioning techniques. (The weak electric fields generated by mormyrids and gymnotoids were not yet known.) According to these reports the highest frequencies which Electrophorus was able to hear were about $1 \mathrm{kHz}$; in mormyrids this limit was found to be near $3 \mathrm{kHz}$. The value for Electrophorus was considered rather low for an ostariophysine species (the cyprinid Phoxinus laevis, e.g., had its upper hearing limit between 


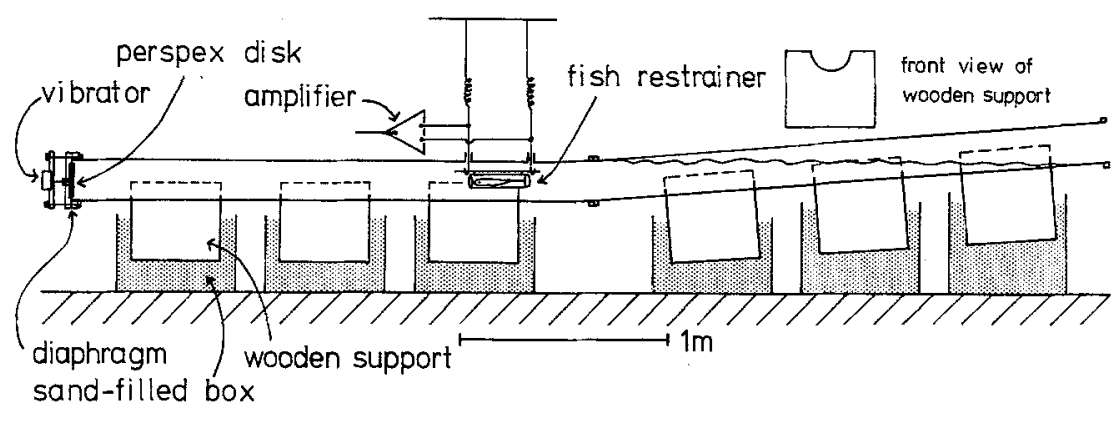

Fig. 1. The apparatus used to stimulate and record the EODs from electric fish. The animal was put in a plastic mesh restrainer which was pulled into the tube and positioned at approx. $200 \mathrm{~cm}$ distance from the diaphragm by two fine threads (one on each side of the restrainer; not shown). The restrainer was supported by the leads of the EOD recording electrodes. The upturned open end of the tube prevented water from flowing out and attenuated standing waves and reflections in the tube (further details: see Material and Methods)

5,000 and $7,000 \mathrm{~Hz}$ ) whereas the upper hearing limit of the mormyrids was surprisingly high for non-ostariophysines. This was explained by the presence of a gas bladder on each side of the skull connected directly with the sacculus which could take over the function of the swim bladder-Weberian ossicles connection of ostariophysines (von Frisch 1936).

Still unclear is the presence of an EOD response to sound in which species (or groups of species) of weakly electric fishes, as well as the exact nature of the EOD responses to sound. Therefore, in this study we report on quantitative stimulus-response relationships and demonstrate that the EOD sound response described below can be used as a tool for investigating the hearing capacities of some pulse-type weakly electric fish.

\section{Materials and Methods}

Weakly Electric Fish Species Used. Gymnotoidei: We used four individuals of Hypopomus sp. 1 (as referred to in Kramer et al. 1981), 2 Gymnotus carapo, 1 Rhamphichthys sp. 3, 1 Apteronotus albifrons, 1 Eigenmannia sp. 3 all from the Solimoes near Manaus, Amazonas, and one individual of another Hypopomus species strongly resembling our H. sp. 2 from Manaus but obtained from a fish dealer (origin unknown). (For taxonomical difficulties with the genera Hypopomus, Rhamphichthys, and Eigenmannia refer to Kramer et al. 1981). Mormyridae: the only species used was Brienomyrus niger ( 2 individuals).

Methods. The fish were tested in a $500 \mathrm{~cm}$ perspex tube (inner diameter: $188 \mathrm{~mm}$; wall thickness: $6 \mathrm{~mm}$ ) placed horizontally on wooden supports in sand-filled boxes to minimize substrate vibrations (Fig. 1). The tube consisted of two parts of equal lengths screwed firmly together. The first part was positioned horizontally, the second part was inclined at an angle of $4.5^{\circ}$ so that the water column slowly decreased to zero at the open end of the inclined part of the tube. The horizontal part of the tube was closed by a strong $(3 \mathrm{~mm})$ rubber sheet on both sides of which two perspex disks (diameter: $15 \mathrm{~cm}$; thickness: $4 \mathrm{~mm}$ each) were concentrically screwed together in a sandwich arrangement. The centre of the outer disk was connected rigidly to the spigot of a moving coil vibrator (Derritron VP2 MM, d.c. to $20 \mathrm{kHz}$ ) the body of which was fixed to a perspex collar mounted on the end of the tube by eight solid bolts. This arrangement allowed the inner disk which faced the water to generate nearly plane sound waves when moved sinusoidally by the vibrator. This was confirmed by measurements of the sound pressure over cross-sections of the tube. The gradually tapering water level in the tilted end of the tube attenuated reflections back into the horizontal part of the tube where the experimental fish was housed. No effort was made to measure the amount of energy reflected at the water-air interface; instead, the sound pressure at the position of the fish was determined.

In the experiment the fish was confined to a specified position approx. in the eenter of the horizontal tube by a small plastic mesh restrainer at a distance of approx. $200 \mathrm{~cm}$ from the sound source. Restrainers of different size were used according to the body sizes of the specimens tested; mesh size was $1.8 \mathrm{~mm}$. The restrainer was held in position by the EOD recording electrodes (fine silver wires) whose leads left the testing tube without touching it by two holes (diameter: $2 \mathrm{~cm}$ ) on top of the tube. The leads were attached to very soft metal springs hanging from an overhead bar, the supports of which were placed in sand-filled boxes. This arrangement minimized disturbance vibrations transmitted directly to the restrainer by the supporting leads. In this experimental set-up the fish's head could be positioned exactly to $\pm 1 \mathrm{~cm}$ in radial and to $\pm 1 \mathrm{~cm}$ in longitudinal direction.

After each experiment the fish was removed and sound pressure was measured by a hydrophone (Brüel \& Kjaer model 8101 ; $0.1 \mathrm{~Hz}-200 \mathrm{kHz}$ ) positioned as exactly as possible (approx. $\pm 2 \mathrm{~cm}$ ) where the fish's head had been. The electronic stimulation chain consisted of a sine wave oscillator including a pulse shaper (University of Konstanz). Total signal duration was $480 \mathrm{~ms}$ (rise/fall times: $40 \mathrm{~ms}$ each; plateau time: $400 \mathrm{~ms}$ ). The output of the signal generator could be attenuated in $1 \mathrm{~dB}$-steps by a Hewlett-Packard model $350 \mathrm{D}$ attenuator and was finally amplified (Hewlett-Paekard model $6824 \mathrm{~A}$ amplifier/power supply; d.c. to $10 \mathrm{kHz}$ ).

A 120-1-aquarium was connected to the testing tube by two flexible plastic tubes (inner diameter: $9 \mathrm{~mm}$ ). In this aquarium water was heated and aerated; when no fish was tested the water of the testing tube was circulated through this aquarium by means of a small aquarium pump. Water temperature ranged from 29.5 to $26^{\circ} \mathrm{C}$.

Stimulation and Recording Procedure. EODs were recorded differentially by two fine silver wire electrodes, one in front of the fish's head and one behind its tail (Fig. 1). A Hewlett-Packard 9825A desktop computer could run a complete experiment and measurement cycle automatically. This included acoustic stimulation at a precisely specified time relative to the EOD activity of the fish, and measuring and recording EOD activity prior to, during and after acoustic stimulation as well as statistical analysis of the fish's response as soon as the measurement sweep was completed. A measurement sweep was started by a start command from the $9825 \mathrm{~A}$ computer to a Nicolet 1074 multi-channel digital analyzer (with models SH-71 and SH-72 plug ins) which measured and stored successive, individual inter-EOD intervals. The sound pulse was triggered on-line to the digital analyzer by one specific EOD 


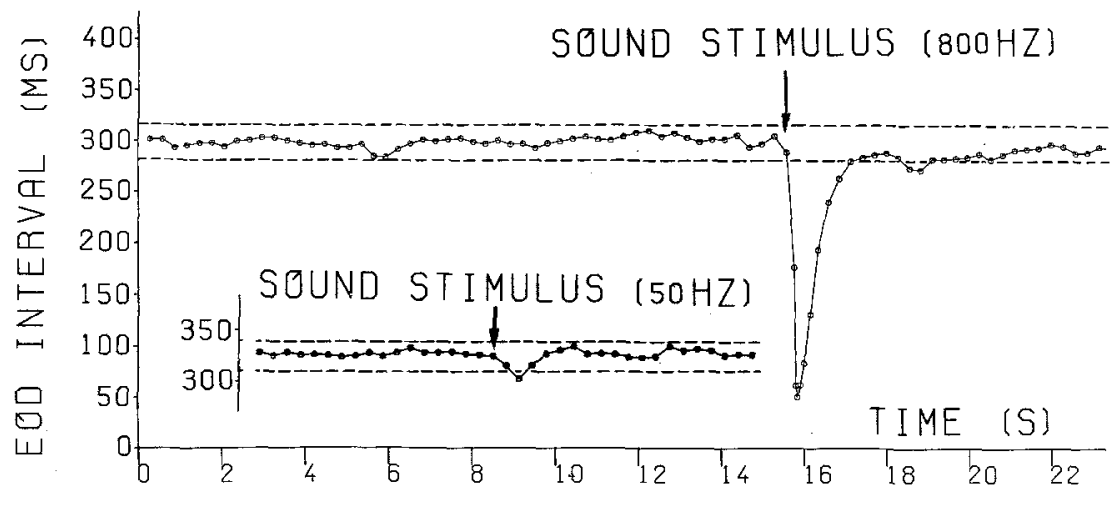

Fig. 2. The EOD sound response in Hypopomus sp. 1. Each point is one EOD; the ordinate shows the EOD interval duration, the abscissa the time of occurrence of each EOD. $800 \mathrm{~Hz}$ stimulus: high sound intensity. Note that the first EOD interval (the interval in which the stimulus occurred) was significantly shortened. Dashed lines indicate the twosided $P<0.001$ confidence limits around the average of the last $52 \mathrm{EOD}$ intervals preceding sound onset (arrow). $50 \mathrm{~Hz}$ stimulus (inset): response near threshold. The second EOD interval after stimulus onset was significantly shortened

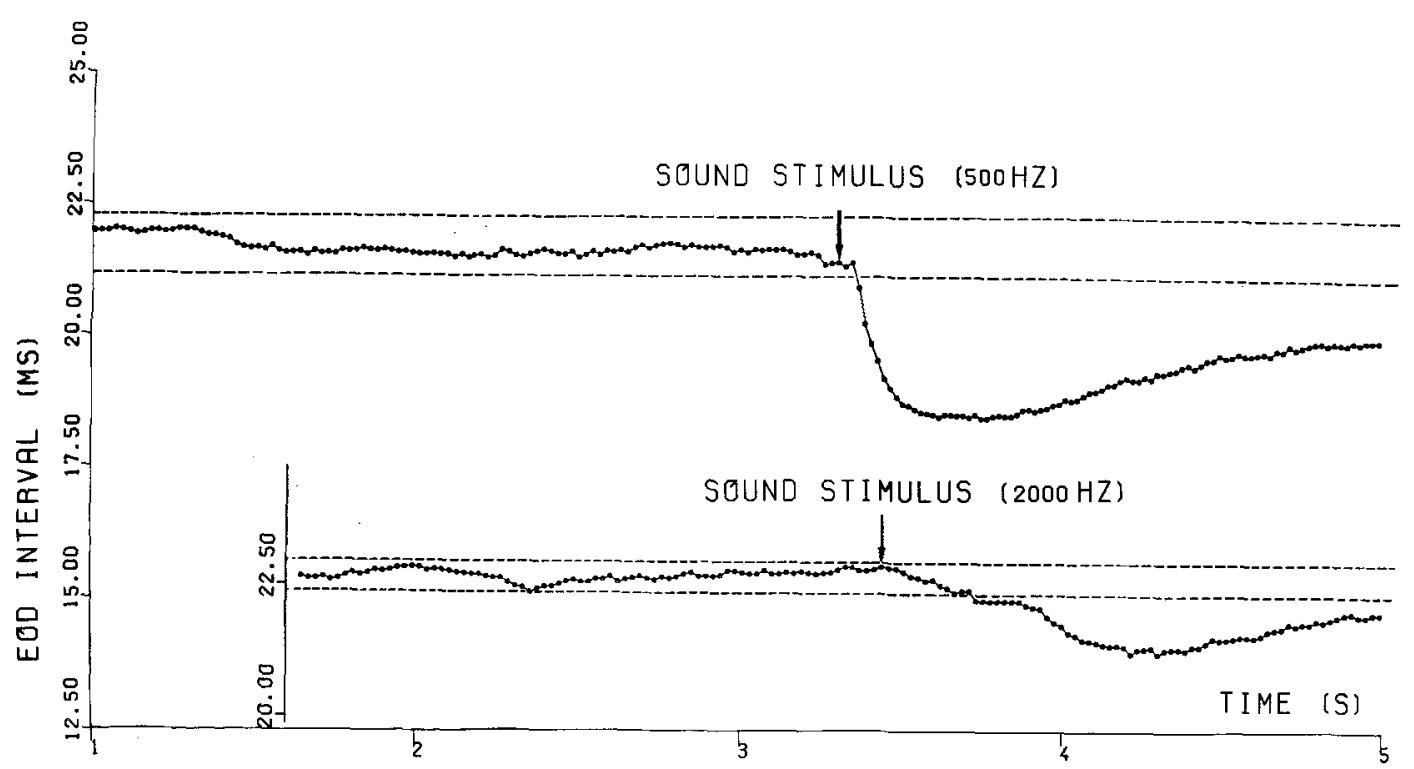

Fig. 3. The EOD sound response in Gymnotus carapo. As Fig. 1. Confidence limits were calculated from the last 152 EOD intervals preceding onset of stimulation (not all shown). Inset (2,000 $\mathrm{Hz}$ stimulus): medium high sound intensity. Note rather long latency of response. $500 \mathrm{~Hz}$ stimulus: high sound intensity. Note very short latency of response and stronger excursion of subsequent EOD intervals to shorter durations

when a preset address number of the analyzer's memory was reached. Plots of the $\mathrm{EOD}$ responses were made by the computer centre of the University of Konstanz.

A response to an acoustic stimulus was considered positive when EOD intervals following stimulus onset were beyond the two-sided $1 \%$ confidence limits of the EOD interval average before stimulation. The number of EOD intervals considered for this average varied from 52 in the low EOD-rate Hypopomus and Brienomyrus, and 152 in Gymnotus to over 400 in Rhamphichthys, Eigenmannia and Apteronotus.

Determination of Thresholds of the EOD Sound Response. The fish were stimulated at a specific sound frequency and intensity. When a response on at least the $1 \%$-significance level was observed, stimulation intensity was decreased in $10 \mathrm{~dB}$-steps until the response disappeared. Then sound intensity was increased in $5 \mathrm{~dB}$ steps until the response reappeared. If no response was elicited by the first stimulus intensity, this was increased in $5 \mathrm{~dB}$-steps until a response could be obtained. Then sound intensity was lowered again in 2 or $3 \mathrm{~dB}$-steps until the response vanished. From this point on stimulation intensity was raised in $1 \mathrm{~dB}$-steps until the threshold response was observed. The next test was performed at least 3 min later. No signs of adaptation of the EOD response to sound was found under these conditions.

\section{Results}

\section{The EOD Response}

Figures 2-4 show EOD responses of the gymnotoids Hypopomus and Gymnotus, and of the mormyrid Brienomyrus to sound stimuli.

Figure 2 (inset) gives a threshold response for $\mathrm{Hy}$ popomus, a low EOD-rate pulser. In this recording, the second EOD interval after stimulus onset was significantly shorter than predicted by the $0.1 \%$-confidence limits surrounding the mean of EOD intervals, as calculated by the last 52 EOD intervals preceding the stimulus. The latency was approx. $600 \mathrm{~ms}$. In 


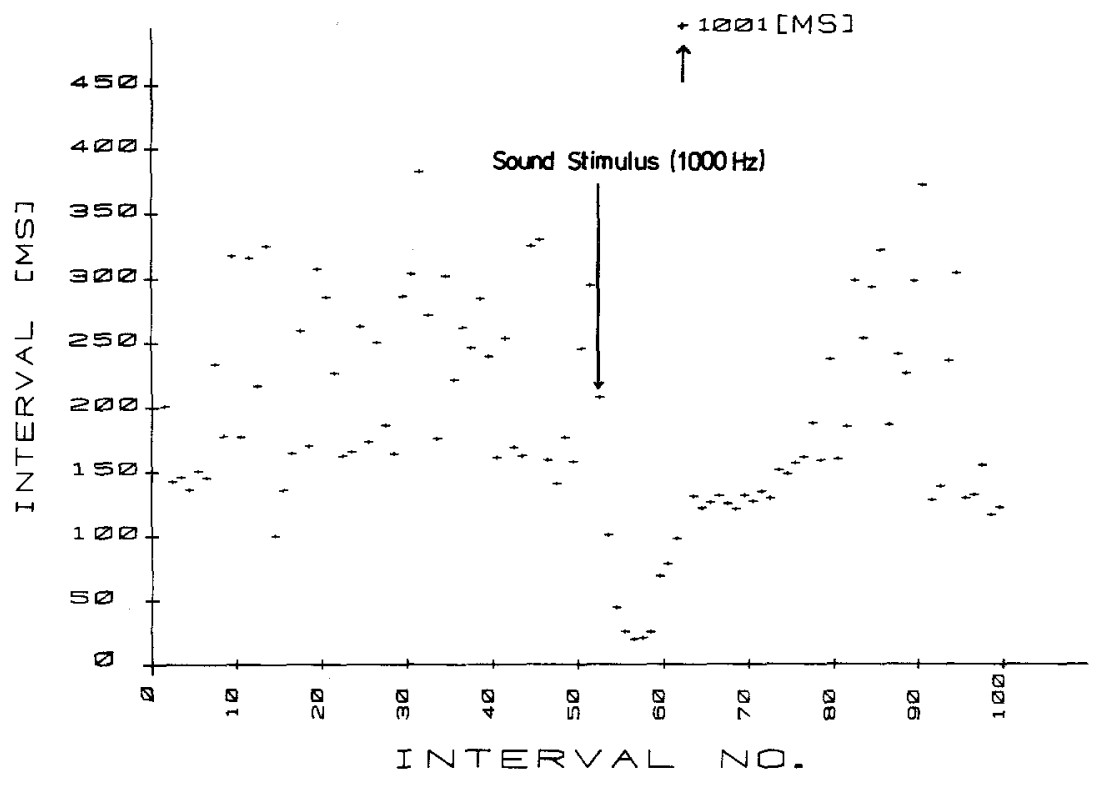

Fig. 4. The EOD sound response in Brienomyrus niger (medium high sound intensity). As Fig. 1 but abscissa shows EOD interval number. Note that the first EOD interval after stimulus onset is shortened. After a transient EOD rate increase an EOD stop of $1 \mathrm{~s}$ occurs in this record

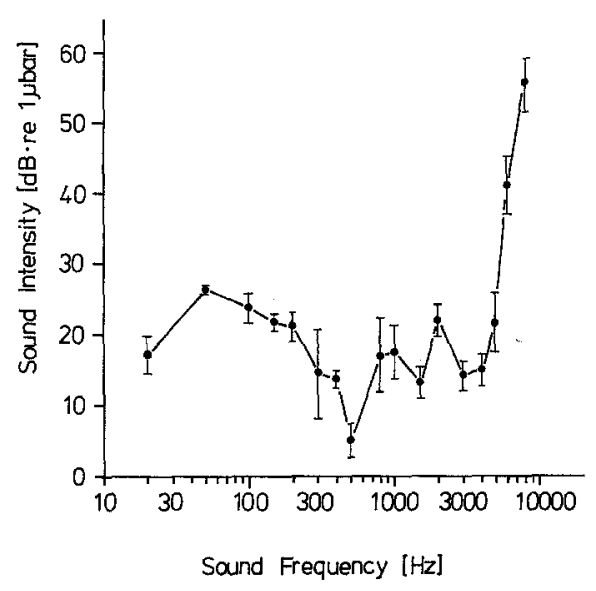

Fig. 5. Thresholds of the EOD sound response to sound of different frequencies (abscissa) in Hypopomus sp. 1. Mean values and \pm 1 standard error ranges for 4 individuals. Ordinate: sound intensity

some cases only the third EOD interval after stimulus onset was affected (latencies approx. $800 \mathrm{~ms}$ to $1200 \mathrm{~ms})$. In by far the most cases, however, already the first interval (whose start coincided with the beginning of the rise time of the sound stimulus) was significantly shorter than the mean. With increasing stimulus amplitude the response generally became stronger and the latency shorter. Whereas in a typical threshold response only one EOD interval was outside the confidence limits this number was considerably greater with stimuli well above threshold. This is also shown in Fig. 2. In this recording, a number of subsequent EOD intervals were still shorter than the first interval, and the animal briefly increased its low EOD rate of approx. $3 \mathrm{~Hz}$ very sharply to approx. $20 \mathrm{~Hz}$.

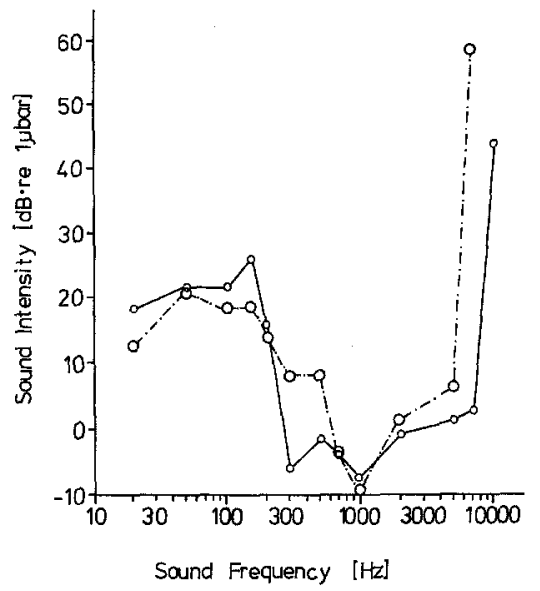

Fig. 6. Thresholds of the EOD sound response to sounds of different frequencies in Gymnotus carapo. As Fig. 5. Two curves for two individuals

Latencies below $200 \mathrm{~ms}$ (as in Fig. 2) were rather rare, but the shortest reaction time observed was $100 \mathrm{~ms}$. Another Hypopomus species strongly resembling $\mathrm{Hy}$ popomus sp. 2 (Kramer et al. 1981) showed very similar responses.

Gymnotus carapo discharges much more rapidly than Hypopomus. In this species similar results were obtained. At threshold stimulus intensities the EOD response was weak and latencies long (>0.5 s) compared to the responses to more intense stimuli (Fig. 3). At high stimulus intensities there were only two intervening EOD intervals which were unaffected (Fig. 3); the shortest latencies were approx. $60 \mathrm{~ms}$. The amplitudes of the transient EOD rate increases were clearly correlated with sound intensity. 


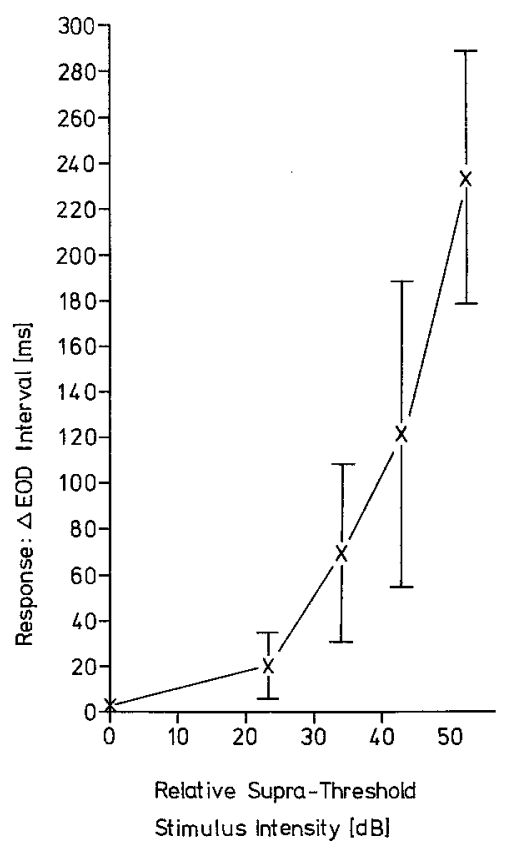

Fig. 7. Intensity-dependence of the EOD sound response in Hypopomus sp. 1. Abscissa: Sound intensity above threshold; ordinate: difference between EOD interval mean before stimulation and the shortest of the three EOD intervals following stimulus onset. Mean values and \pm 1 standard deviation ranges are shown for 4 responses at each point (one individual). Sound frequency: $150 \mathrm{~Hz}$

In Brienomyrus the response also appeared graded with stimulus intensity. This was more difficult to demonstrate here since in this species, as in other mormyrids, resting discharge rate exhibits a more complicated pattern (Kramer 1976) than in gymnotoids. Even the first interval was shortened (Fig. 4) by a medium intensity stimulus delivered during low EOD rate.

No response to sound of even the highest available intensities at all tested frequencies $(20-10,000 \mathrm{~Hz} ; 50$ $60 \mathrm{~dB}$ re: $1 \mu \mathrm{bar}$ ) was obtained in Rhamphichthys, and the two wave EOD species Eigenmannia and Apteronotus. Although in Rhamphichthys the $1 \mu$ s resolution of the digital clock used corresponds to an EOD interval measurement precise roughly to the nearest $1 /$ 16,000 th (or $0.006 \%$ ) no acceleration or deceleration of EOD rate in response to sound, however small, was detected.

\section{Frequency Dependence of the EOD Sound Response}

Four individuals of Hypopomus sp. 1 and two individuals of Gymnotus were tested for frequency-dependence of the threshold of the EOD sound response (Figs. 5, 6). The results in both species were rather similar although Gymnotus appeared more sensitive in absolute terms and had its maximum sensitivity at a higher frequency $(1,000 \mathrm{~Hz}$ at ca. $-9 \mathrm{~dB}$ re: $1 \mu \mathrm{bar}$ instead of $500 \mathrm{~Hz}$ at ca. $5 \mathrm{~dB}$ re: $1 \mu$ bar in
Hypopomus). Both species responded well at all frequencies up to $5,000 \mathrm{~Hz}$ (one individual of Gymnotus even to $7,000 \mathrm{~Hz}$ ).

On the low frequency end, thresholds rose rather steeply between approx. $300 \mathrm{~Hz}$ and $100 \mathrm{~Hz}$; below this frequency thresholds changed little and even began to drop again below $50 \mathrm{~Hz}$. Due to limitations of the experimental setup and to higher amplifier background noise level between approx. 40 and $170 \mathrm{~Hz}$ we regard the threshold values below $400 \mathrm{~Hz}$ as less reliable than those at higher frequencies. Below ca. $360 \mathrm{~Hz}$ it has to be assumed that near-field effects (high particle velocity) increased in importance (Banner 1967); no effort was made to measure the particle velocity (Veit 1977; Tautz 1979) to pressure ratio. This is planned for future investigations.

\section{Intensity Dependence of the EOD Sound Response}

Figure 7 shows the relationship between intensity of sound $(150 \mathrm{~Hz})$ and amplitude of EOD response in Hypopomus measured as the difference between mean EOD interval duration before stimulus onset and the shortest EOD interval within the three intervals immediately following stimulus onset (cf. Fig. 2). The EOD response appears graded with stimulus intensity in sigmoid fashion; response saturation was not achieved at available intensities.

\section{Discussion}

\section{Characteristics of the EOD Sound Response}

This study demonstrates that the EOD rate of some pulse type weakly electric fish can be influenced by sound stimuli in a highly predictable fashion, and that this response can be used to investigate the auditory capacities in those fish. The EOD sound response in Hypopomus, Gymnotus and Brienomyrus has reflexlike properties: it exhibits a well-defined time relationship to the stimulus with little or no adaptation. The response was graded with amplitude.

The EOD sound response can be particularly useful for investigations of fish hearing for two reasons: (1) no conditioning or other treatments prior to testing are needed, (2) the decision whether a response was positive or not can be made with exceptional precision by computer according to predetermined criteria, excluding an observer's bias.

\section{How Reliable Are the Threshold Determinations?}

Due to technical limitations we may not have been able to measure absolute thresholds of the EOD sound response (especially at low frequencies). It 
should, however, be kept in mind that we measured thresholds of the unconditioned EOD sound response which may not at all be identical with hearing thresholds. Using conditioning techniques (usually more suitable to determine absolute auditory thresholds, see Markl and Ehret 1973) the majority of studies report auditory thresholds between 300 to $1,000 \mathrm{~Hz}$ at -40 to $-45 \mathrm{~dB}$ re: $1 \mu$ bar in ostariophysine species (cf. reviews in Popper and Fay 1973; Tavolga 1976). Depending on stimulation as well as measurement parameters, threshold determinations in the goldfish varied up to $70 \mathrm{~dB}$ (cf. Popper and Fay 1973). Our threshold values compare, e.g., with the auditory thresholds in the ostariophysine Leucaspius delineatus (Schade 1971) which were criticized for being too high (Tavolga 1976). At present the possibility cannot be ruled out that our thresholds are relative values which would be lower under different acoustic conditions ('pure' sound pressure field: Hawkins and MacLennan 1976; lowest possible ambient noise level: Tavolga 1967; Buerkle 1968; Hawkins and Chapman 1975).

Most importantly, the frequencies of greatest sensitivity (close to $1,000 \mathrm{~Hz}$ in Gymnotus and close to $500 \mathrm{~Hz}$ in Hypopomus) agree well with the range of best hearing as demonstrated for other ostariophysine fish. Also the upper response limits (at least $5,000 \mathrm{~Hz}$ in Hypopomus and at least 7,000 $\mathrm{Hz}$ in one Gymnotus individual) agree well with upper hearing limits found in ostariophysines (Popper and Fay 1973).

\section{Species Divergence in the EOD Sound Response}

Wave fishes (Eigenmannia and Apteronotus) as well as the pulse fish Rhamphichthys did not show the slightest indication of an EOD sound response even to the highest intensities our stimulation equipment was able to deliver.

It appears that probably all gymnotoids where increased motor activity is correlated with an increase in EOD rate (Hypopomus sp. 1 and sp. 2; Gymnotus carapo, Black-Cleworth 1970), and which change EOD rate in response to vibration, light, touch, or food do display the EOD sound response. Those species where EOD rate is independent of ongoing motor activity and external stimulation, except narrowly defined electrical stimulation (review in Heiligenberg 1977), i.e., probably all wave species and some pulse species as Rhamphichthys (cf. Scheich et al. 1977), very likely do not possess the EOD sound response.

An explanation of this divergence of species with respect to the presence of the EOD sound response might be that in those species where increased motor activity, and external stimulation, is correlated with increased EOD rate, a connection between motor behaviour command centre(s) and the electromotor system still exists (probably including the nucleus magnocellularis mesencephali, described by Szabo 1967; Réthelyi and Szabo 1973). So any sensory input modifying the state of excitation might influence the electromotor system via the motor behaviour command centre(s). In wave fishes and certain gymnotoid pulse species the electromotor system apparently has become emancipated from motor behaviour command control, and sensory input can no more directly influence the electromotor system (except electrical stimulation).

If this hypothesis were correct then the EOD sound response, where it exists, should not differ from EOD responses to light, touch, vibration, and sudden changes in temperature and odour (Jäger 1974). One of the three 'immediate' types of responses to the onset of electrical stimulation obtained in Gymnotus (Westby 1975), the 'frequency increase', is indistinguishable from the EOD sound response in Gymnotus presented here. Also the shortest latencies to sound and to electrical stimuli were similar, somewhat below $70 \mathrm{~ms}$ and $50 \mathrm{~ms}$, respectively, with two intervening unaffected intervals in both cases. The slight difference in minimum reaction time can be explained by different EOD resting rates, and by the gradual rise of intensity of our sound stimulus.

Input from electroreceptors is relayed to the midbrain via the fast electrosensory system at only 0.8 $1.5 \mathrm{~ms}$ latency (Schlegel 1977). Conduction time of acoustic input to the midbrain is only slightly longer (3.5-4.3 ms at optimal stimulus intensities, Grözinger 1967). This similarity points to the possibility that a great part of the presumed multi-synaptic pathway, linking the nucleus magnocellularis mesencephali with the medullary pacemaker (see also Schlegel 1977), may equally be involved in the EOD sound response in Gymnotus.

One may ask what (if any) biological significance the transient EOD rate increase to sound has. An EOD rate increase improves temporal resolution for changes in the environment (Hagiwara and Morita 1963). A useful information increase occurs up to an EOD rate of approx. $80 \mathrm{~Hz}$ (Schlegel 1973; as cited by Westby 1975). However, several gymnotoid pulse species discharging at only somewhat higher EOD resting rates compared to Gymnotus (such as at least one Rhamphichthys species, Steatogenys elegans and Hypopygus sp.; $55-65 \mathrm{~Hz}$, cf. Kramer et al. 1981) lack or probably lack the response.

Biological functions of hearing in fish have quite recently been discussed in Schwartzkopff (1976). Acoustic communication by sound signals (apart from sounds generated by movements; Piddington 1972) seems unlikely in gymnotoids but has to be 
considered in mormyrids. Sounds produced during agonistic encounters with conspecifics were reported in G. petersii (Rigley and Marshall 1973). We plan to investigate such sound production in greater detail.

We wish to thank Dr. J. Kien (University of Regensburg), Dr. F. Kirschbaum (University of Köln), and Dr. G.W.M. Westby (University of Sheffield) for critically reading the manuscript. This work was supported by the Deutsche Forschungsgemeinschaft (grants $\mathrm{Kr} \mathrm{446/8,} \mathrm{Ma} \mathrm{374/10).} \mathrm{We} \mathrm{wish} \mathrm{to} \mathrm{thank} \mathrm{the} \mathrm{computer}$ centre and the mechanical, the electronical, and the carpentry workshop of the University of Konstanz for excellent aid. It is a pleasure to acknowledge the expert technical assistance of H.-J. Burger.

\section{References}

Banner A (1967) Evidence of sensitivity to acoustic displacements in the lemon shark. In: Cahn P (ed) Lateral line detectors. Indiana University Press, Bloomington, pp 265-273

Bauer R (1974) Electric organ discharge activity of resting and stimulated Gnathonemus petersii (Mormyridae). Behaviour 50:306-323

Black-Cleworth P (1970) The role of electrical discharges in the non-reproductive social behavior of Gymnotus carapo L. (Gymnotidae, Pisces). Anim Behav Monogr 3:1-77

Boutteville $\mathrm{K}$ von (1935) Untersuchungen über den Gehörsinn bei Characiniden und Gymnotiden und den Bau ihres Labyrinthes. Z Vergl Physiol 22:162-191

Buerkle U (1968) Relations of pure tone thresholds to background noise level in the Atlantic cod Gadus morhua. J Fish Res Board Can 25:1150-1160

Diesselhorst $G$ (1938) Hörversuche an Fischen ohne Weberschen Apparat. Z Vergl Physiol 25:748-783

Frisch K von (1936) Über den Gehörsinn der Fische. Biol Rev $11: 210-246$

Frisch K von (1938) The sense of hearing in fish. Nature 141:8-11

Grözinger B (1967) Elektro-physiologische Untersuchungen an der Hörbahn der Schleie (Tinca tinca (L.). Z Vergl Physiol 57:44-76

Hagiwara S, Morita H (1963) Coding mechanisms of electroreceptor fibers in some electric fish. J Neurophysiol 25:430-449

Hawkins AD (1973) The sensitivity of fish to sounds. Oceanogr Mar Biol Annu Rev 11:291-340

Hawkins AD, Chapman CJ (1975) Masked auditory thresholds in the cod, Gadus morhua L. J Comp Physiol 103:209-226

Hawkins AD, MacLennan DN (1976) An acoustic tank for hearing studies on fish. In: Schuijf A, Hawkins AD (eds) Sound reception in fish. Elsevier, Amsterdam Oxford New York, pp 149 169

Heiligenberg WF (1977) Principles of electrolocation and jamming avoidance in electric fish. Studies of brain function, vol 1. Springer, Berlin Heidelberg New York, pp 1-85

Jäger U (1974) Geruchsrezeption und Entladungsaktivität bei dem schwachelektrischen Fisch Gnathonemus petersii (Günther 1862) (Mormyridae, Teleostei). Diss Math-Nat Fak Univ Saarland, Saarbrücken (FRG)

Kramer B (1976) The attack frequency of Gnathonemus petersii towards electrically silent (denervated) and intact conspecifics, and towards another mormyrid (Brienomyrus niger). Behav Ecol Sociobiol $1: 425-446$

\section{Note Added in Proof}

Other Rhamphichthys species may well possess the EOD sound response as our Rhamphichthys sp. I (cf. Kramer et al. 1981) showed EOD-rate changes related to motor behaviour.
Kramer B, Kirschbaum F, Markl H (1981) Species specificity of electric organ discharges in a sympatric group of gymnotoid fish from Manaus (Amazonas). In: Szabo T, Czéh G (eds) Sensory physiology of lower aquatic vertebrates. Adv Physiol Sci, vol 31. Pergamon Press/Akademiai Kiadó, Budapest, pp 195-219

Lissmann HW (1958) On the function and evolution of electric organs in fish. J Exp Biol 35:156-191

Lissmann HW (1961) Ecological studies on gymnotids. In: Chagas C, Paes de Carvalho A (eds) Bioelectrogenesis. Elsevier, Amsterdam Oxford New York, pp 215-226

Markl H, Ehret G (1973) Die Hörschwelle der Maus (Mus musculus). Eine kritische Wertung der Methoden zur Bestimmung der Hörschwelle eines Säugetieres. Z Tierpsychol 33:274-286

Piddington RW (1972) Auditory discrimination between compressions and rarefactions by goldfish. J Exp Biol 56:403-419

Popper AN, Fay RR (1973) Sound detection and processing by teleost fishes: a critical review. J Acoust Soc Am 53:1515-1529

Réthelyi M, Szabo T (1973) A particular nucleus in the mesencephalon of a weakly electric fish, Gymnotus carapo (Gymnotidae). 1. Light microscopic structure. Exp Brain Res 17:229-241

Rigley L, Marshall JA (1973) Sound production by the elephant nose fish, Gnathonemus petersii (Pisces, Mormyridae). Copeia 1973(1):134-136

Schade R (1971) Experimentelle Untersuchungen zum Hörvermögen an Leucaspius delineatus. Biol Zentralbl 90:337-356

Scheich H, Gottschalk B, Nickel B (1977) The jamming avoidance response in Rhamphichthys rostratus: An alternative principle of time domain analysis in electric fish. Exp Brain Res 28:229233

Schlegel PA (1977) Electroreceptive single units in the mesencephalic magnocellular nucleus of the weakly electric fish Gymnotus carapo. Exp Brain Res 29:201-218

Schwartzkopff $J$ (1976) Comparative-physiological problems of hearing in fish. In: Schuijf A, Hawkins AD (eds) Sound reception in fish. Elsevier, Amsterdam Oxford New York, pp 3-17

Stipetić E (1939) Über das Gehörorgan der Mormyriden. Z Vergl Physiol 26:740-752

Szabo T (1967) Activity of peripheral and central neurons involved in electroreception. In: Cahn P (ed) Lateral line detectors. Univ Indiana Press, Bloomington, pp 295-311

Tautz J (1979) Reception of particle oscillation in a medium an unorthodox sensory capacity. Naturwissenschaften $66: 452-$ 461

Tavolga WN (1967) Masked auditory thresholds in teleost fishes. In: Tavolga WN (ed) Marine bio-acoustics, vol 2. Pergamon Press, Oxford New York, pp 233-245

Tavolga WN (1971) Sound production and detection. In: Hoar WS, Randall DJ (eds) Fish physiology, vol 5. Academic Press, New York, pp 135-205

Tavolga WN (1976) Recent advances in the study of fish audition. In: Tavolga WN (ed) Sound reception in fishes. Benchmark papers in animal behavior, vol 7. Dowden, Hutchinson and Ross, Stroudsberg, Philadelphia, pp 37-52

Veit I (1977) Ein kleines Druckgradientenhydrofon zur Messung der Schallschnelle in Flüssigkeiten. Akustika 38:72-75

Westby GWM (1975) Has the latency-dependent response of Gymnotus carapo to discharge-triggered stimuli a bearing on electric fish communication? J Comp Physiol 96:307-341 